\title{
A SHARP INEQUALITY AND THE INRADIUS CONJECTURE
}

\author{
FARUK F. ABI-KHUZAM AND ROY BARBARA
}

Abstract. We supply a proof of the following statement: The inradius of a triangle contained inside a (closed) unit square never exceeds $\frac{\sqrt{5}-1}{4}$.

Mathematics subject classification (2000): 51M16.

Key words and phrases: Triangle, inradius, inequality.

\section{REFERENCES}

[1] CoXeter, H. S. M. AND GReitzer S. L., Geometry Revisited, Washington: Math. Assn. America, 1967.

[2] FunAR, L., “Problem 6477”, Amer. Math. Monthly 81 (1984), 588.

[3] Mitrinovic D. S., PeCARIC J. E., VoleneC V., Recent Advances In Geometric Inequalities, Kluwer Academic Publishers, 1989. 